\title{
INVESTIGATION OF FAN FAULT PROBLEMS USING VIBRATION AND NOISE ANALYSIS
}

\author{
Selçuk ERKAYA \\ Mechatronics Engineering Department \\ Erciyes University, Engineering Faculty \\ Kayseri, Turkey \\ serkaya@erciyes.edu.tr
}

\author{
Şaban ULUS \\ Mechatronics Engineering Department \\ Erciyes University, Engineering Faculty \\ Kayseri, Turkey \\ sabanulus@erciyes.edu.tr
}

\begin{abstract}
Cooling fans are used to solve the thermal problems of most critical electronic parts in systems. Therefore, it is very important to monitor the fans condition periodically dealing with the detection, location and analysis of the possible faults. The fault diagnosis of rotating systems is increasingly paying attention. The main purpose of this study is to investigate the possible faults in a rotating system using vibration and noise measurements. A simple cooling fan is used for implementing the experimental measurement. Some artificial faults are performed for measuring the system responses. The empirical results show that Acoustic Emission (AE) technique is very useful to detect the faults at the system.
\end{abstract}

Keywords - Fault detection, vibration and noise measurement, cooling fan, condition monitoring.

\section{INTRODUCTION}

In the todays industry and industrial facility management [1], meeting the consumer needs, continuity of production and safety of the processes have a great importance. Continuous Fault Condition Monitoring (FCM) can avoid downtime and also reduce the total cost of products. In spite of their initial capital investment cost, monitoring the health of these structures has a great importance and FCM needs in the industry is increasing day by day. Providing an early detection of structural, mechanical or electronical problems allow operators to see where faults occurred and suggest when the system will break down approximately. Any abnormalities or faults in the machinery or equipment must be detected and analyzed at the early stage to avoid major problems. Therefore, FCM of rotating machinery has a crucial role in industry as it keeps the system at healthy condition for maximum productivity, while detecting and diagnosing the faults at early stage. As a result, it is possible to prevent the serious problems, damage and more cost.

Tian et al. [2] introduced an approximate theory to describe fan conditions. For the case of different working conditions, vibration signals of the fan were analyzed by utilizing the approximate theory. The obtained results showed that the approximate theory was able to identify the conditions of the fan with faults compared with the normal condition. Miao et al. [3] proposed a vibration based fan bearing fault detection through the wavelet transform and the Hilbert transform in computer cooling fan systems.

An experiment study was implemented to identify the different bearing faults. Elmaleeh et al. [4] applied several condition monitoring techniques to improve the plant reliability and reduce the downtime. Effectiveness of $\mathrm{AE}$ technique was investigated for incipient detection of faults at rotating machineries. The exact faults in the machine were tried to identify using time and frequency analysis of the signals. Velarde-Suarez et al. [5] employed an experimental study about the aerodynamic tonal noise sources in a centrifugal fan with backward-curved blades.

Acoustic and pressure fluctuation measurements were implemented for different flow rates. For explaining the some features of the aerodynamic tonal noise generation, both pressure and vibration signals were analyzed and correlation analysis between methods was discussed. Another study of the same research group [6] investigated the influence of some geometric features of the aero acoustic behavior of a squirrelcage fan, used in automotive air conditioning units. They focused on the effect of both shape and the position of the volute tongue on the noise generated by the fan. Acoustic behavior of the fan was characterized by acoustic pressure measurements near the fan inlet.

Frequency analysis results were used to describe the characteristics of the fan. Wu et al. [7] presented a formula for predicting the noise spectra of axial flow fans. Acoustic pressure and line spectrum were obtained by taking a Fourier series expansion and also, a normal distribution like shape function was designed which divided the frequency into consecutive bands at BPF and its harmonics. Lin et al. [8] performed an experimental study for monitoring the possible faults using AE technique based on Hilbert-Huang transform. Time-frequency analysis was used for extracting the features of the wind turbine bearings. $\mathrm{AE}$ in the wind turbine bearing was described in terms of features such as frequency and energy. Tian [9] employed a study based on the major failure mechanisms and failure modes of cooling fan system. An accelerated life testing methodology was presented. Reliability analysis and failure criteria of components were analyzed and life testing was accelerated by using high temperature testing, 
condition monitoring, noise control, failure mode analysis and Weibull distribution. Yin et al. [10] proposed a fan system condition monitoring based on virtual prototyping technology. Three dimensional model of the fan system was built and imported to the ADAMS to simulate the system under different working conditions.

Measured and virtual signal data were obtained and compared to identify the real fan condition. Three accelerometers were used to obtain real vibration signal data. For pattern recognition, cross correlation function was selected to find the most similar simulation condition. Tandon ve Choudhury [11] tried to describe the source of vibration and noise generation on bearings. Using the signal processing techniques in time and frequency domain, the high frequency resonance technique was applied. On the other hand, sound pressure, sound density and sound radiation techniques were used for acoustic measurements. Waveform transforming and automatic data processing techniques were used to detect bearings faults. Loutas et al. [12] implemented some practices using acoustic emission techniques for detecting faults on rotary machine elements such as gearboxes. AE signals of the healthy gearbox were identified and related data were obtained by creating artificial failures. Toutountzakis et al. [13] applied $\mathrm{AE}$ techniques to detect the possible faults at rotating machinery elements such as gearboxes and practiced to determine fault characteristics of seeded gearboxes. As a result, it was observed that $\mathrm{AE}$ technique had some application difficulties in which some seeded artificial fault locations.

Cooling fans are one of the most critical parts in computer system and in most of electronic products. Since a fan assembly includes both electronic and mechanical parts, it can fail electronically, mechanically, or both. The electronic parts include control circuitry, motor and its mechanical and electronic components [14]. The mechanical parts of a fan consist of bearing, lubricant, shaft, fan blades or propeller and fan housing. A fan can have either "hard failures" in which the fan is non-functional or "soft failures" (parametric failures) such as slower RPM, increased input current, or higher noise level [9]. To have a good experience of fan reliability, all possible failure mechanisms and failure modes of the components should be considered. Cooling fans have a great importance in many electronic systems. Researches performed up to now, have been usually focused on these techniques such as classical vibration monitoring technique, thermal analysis, corrosion and performance analysis technique, analyzing oil particle properties. In addition to that, in recent years, AE technique has become the focus of attention by the researchers. AE is defined as the elastic waves which results of rapid release of the energy in many localized sources of materials under stressed conditions. At the researches, they pointed to many advantages of $\mathrm{AE}$ technique such as, earlier fault detection, detecting micro cracks and wear. This technique is based on monitoring the systems at their working conditions for detecting the possible faults in mechanical systems before them break down and providing maintenance against these faults.
Main goal of this research is to show the effectiveness of AE technique compared to vibration signal analysis and especially is to show that how AE fault characteristics of different fan faults, such as broken fan blades, deformed blade shape, bearing faults, cracked part at the root of the fan blades. It is aimed to determine the possible faults that may occur in the system by the acoustic properties obtained from the system. Furthermore, acoustic and vibration characteristics are analyzed together and these characteristics, which are obtained from the system simultaneously, are compared to each other. For this purpose, this paper is organized as follows; Section 2 describes the experimental analysis. Results and conclusion are given in sections 3 and 4, respectively.

\section{EXPERIMENTAL TEST RIG}

In this study, a simple cooling fan in a computer power supply is used for implementing the experimental measurement. The used fan has got seven blades. Artificial fan faults such as broken fan blades, deformed blade shape, bearing faults, lubrication problems, cracked part at the root of the fan blades are considered as outlined in Fig. 1. Both vibration and acoustic measurements are performed to evaluate the system responses.
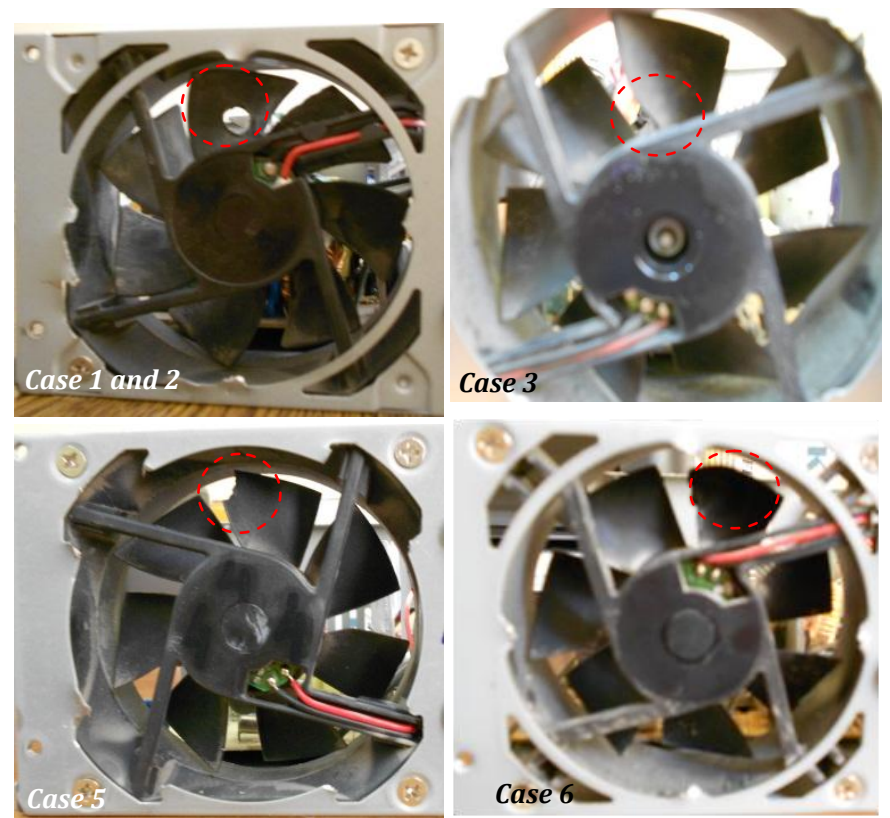

Fig. 1: Artificial fan faults

Classification of the artificial faults is outlined in Table 1.

Table 1. Artificial fault classifications

\begin{tabular}{|l|l|}
\hline Case identification & Fault Description \\
\hline Case 1 & Hole with $4 \mathrm{~mm}$ \\
\hline Case 2 & Hole with $7 \mathrm{~mm}$ \\
\hline Case 3 & Blade fault (near the bearing) \\
\hline
\end{tabular}




\begin{tabular}{|l|l|}
\hline Case 4 & Bearing fault (Lubrication problem) \\
\hline Case 5 & Blade fault \\
\hline Case 6 & Blade shape deformation \\
\hline
\end{tabular}

In the measuring stage, a tool for measuring the vibration and acoustic signals, "Bruel-Kjaer $(\mathrm{B} \& \mathrm{~K})$ portable and multichannel pulse 3560-B-X05" was used to obtain and analyze results. Vibration and acoustic signals were measured simultaneously. Available analysis program is able to measure the time and frequency domain analysis of the obtained signals from the computer cooling fan.

In order to measure the acoustic signals, it was used an acoustic sensor with a preamplifier (4189-A-021 - 1/2inch freefield microphone with Type 2671 preamplifier) and used an accelerometer (B\&K 4514B) for measuring vibration data. Properties of used sensors are outlined in Table 2.

Table 2. Specifications of vibration and acoustic sensors [15]

\begin{tabular}{|c|c|c|c|}
\hline \multicolumn{2}{|c|}{$\begin{array}{l}\text { Properties of acoustic } \\
\text { sensor }\end{array}$} & \multicolumn{2}{|c|}{ Properties of vibration sensor } \\
\hline Diameter & $1 / 2$ inch & Frequency & $\begin{array}{l}1-12000 \\
\mathrm{~Hz}\end{array}$ \\
\hline Dyn. Range & $\begin{array}{l}16.5-134 \\
\mathrm{~dB}\end{array}$ & Sensitivity & $10 \mathrm{mV} / \mathrm{g}$ \\
\hline Freq. Range & $\begin{array}{l}20-20000 \\
\mathrm{~Hz}\end{array}$ & Temperature & $\begin{array}{lll}-51 & \\
121^{\circ} \mathrm{C} \\
\end{array}$ \\
\hline Inherent Noise & $16.5 \mathrm{~dB} A$ & $\begin{array}{l}\text { Residual Noise Level in } \\
\text { Spec Freq Range (rms) }\end{array}$ & $\pm 0.8 \mathrm{mg}$ \\
\hline $\begin{array}{l}\text { Lower Limiting } \\
\text { Frequency }\end{array}$ & $20 \mathrm{~Hz}$ & $\begin{array}{l}\text { Maximum Operational } \\
\text { Level (peak) }\end{array}$ & $500 \mathrm{~g}$ \\
\hline $\begin{array}{l}\text { Pressure } \\
\text { Coefficient }\end{array}$ & $\begin{array}{l}-0.01 \\
\mathrm{~dB} / \mathrm{kPa}\end{array}$ & $\begin{array}{l}\text { Maximum Shock Level } \\
( \pm \text { peak })\end{array}$ & $3000 \mathrm{~g}$ \\
\hline Sensitivity & $50 \mathrm{mV} / \mathrm{Pa}$ & Weight & 8.7 gram \\
\hline Standards & \begin{tabular}{|l|} 
IEC \\
$61094-4$ \\
WS2F \\
\end{tabular} & Electrical Connector & $\begin{array}{l}10-32 \\
\text { UNF }\end{array}$ \\
\hline $\begin{array}{l}\text { Temperature } \\
\text { Coefficient }\end{array}$ & $\begin{array}{l}-0.006 \\
\mathrm{~dB} /{ }^{\circ} \mathrm{C} \\
\end{array}$ & Mounting & Magnet \\
\hline $\begin{array}{l}\text { Temperature } \\
\text { Range }\end{array}$ & $\begin{array}{|ll|}-30 & -100 \\
{ }^{\circ} \mathrm{C} & \\
\end{array}$ & $\begin{array}{l}\text { Clip/Stud/Screw } \\
\text { included }\end{array}$ & $\begin{array}{l}10-32 \\
\text { Stud }\end{array}$ \\
\hline Input Type & $\begin{array}{l}\text { CCLD } \\
\text { IEPE }\end{array}$ & Resonance Frequency & $32 \mathrm{kHz}$ \\
\hline
\end{tabular}
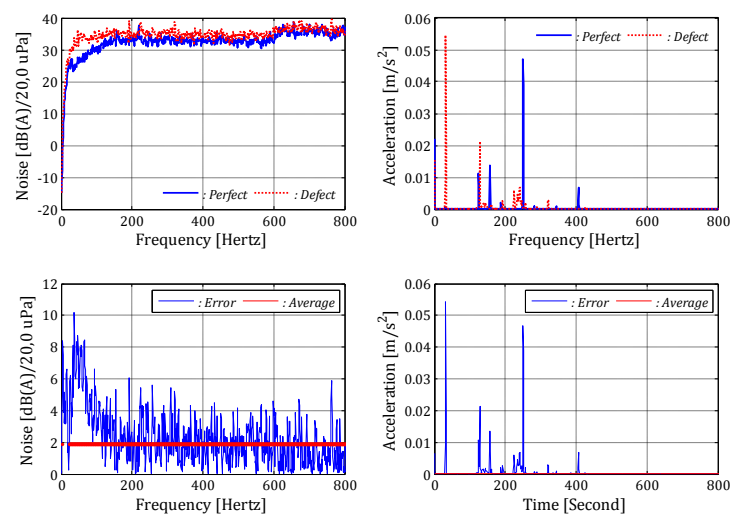

Fig. 3: Noise and vibration measurements for Case 1

In Case 1, an artificial hole with $4 \mathrm{~mm}$ diameter was implemented. Naturally, this fault affects the balancing of the fan. Acoustic and vibration measurements give the differences between healthy and defective fans. When the hole diameter is increased from $4 \mathrm{~mm}$ to $7 \mathrm{~mm}$, the obtained result are outlined in Fig. 4,

Block diagram for vibration and acoustic measurements are outlined in Fig. 2.

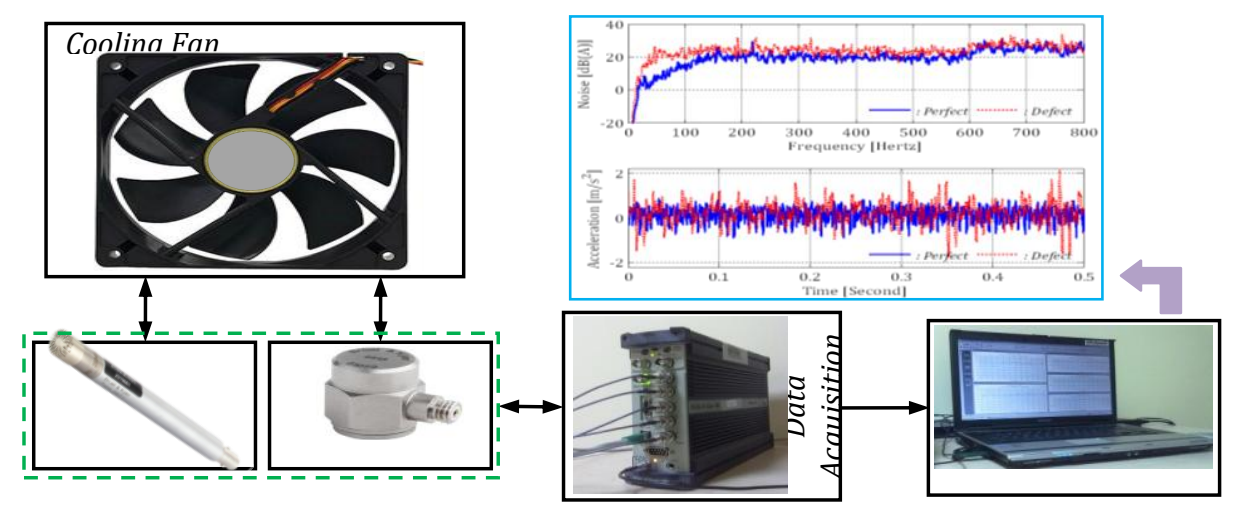



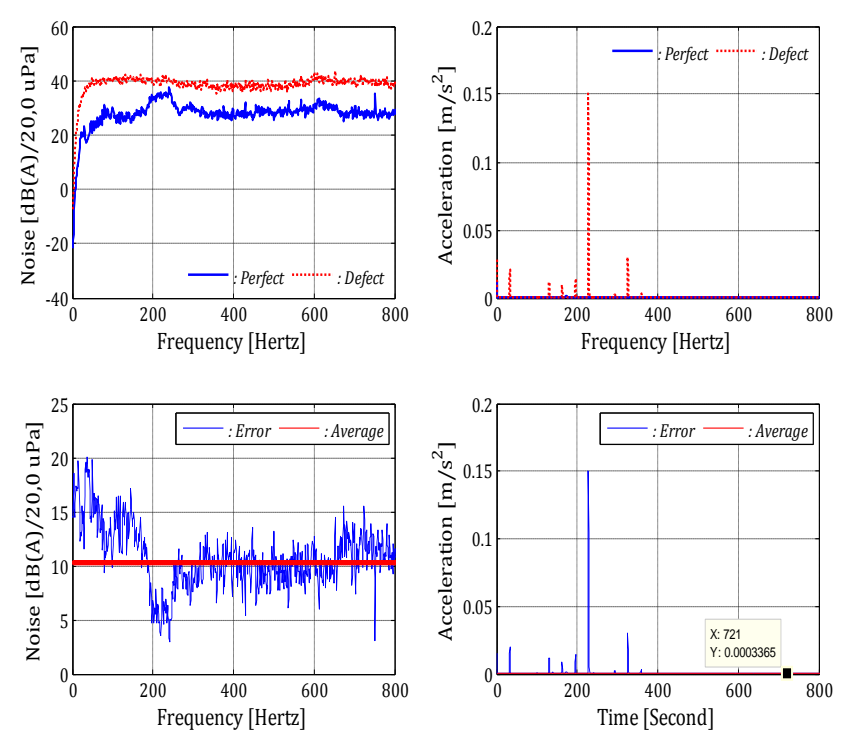

Fig. 4: Noise and vibration measurements for Case 2

It is clear from Fig. 4 that the increasing of hole diameter makes the vibration and noise characteristics worse. Both vibration amplitude and noise level increase. The results of noise and vibration measurements for Case 3 are outlined in Fig. 5,
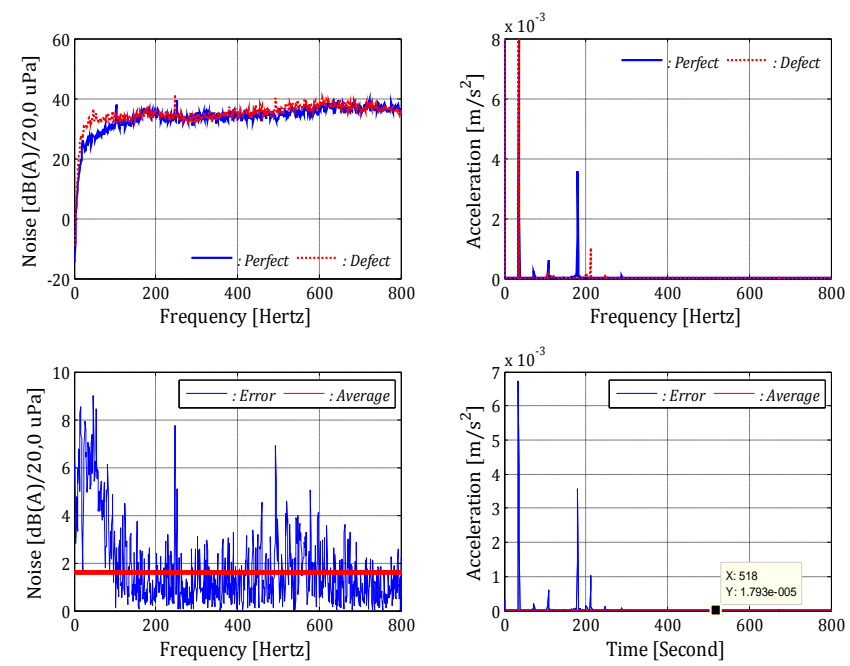

Fig. 5: Noise and vibration measurements for Case 3

As seen from Fig. 5, it is clear that, there is a certain difference between perfect and defect fans. Particularly, noise characteristic has a clear difference. So, it is said that noise measurement can be used effectively to detect this fault type in mechanical systems. For the Case 4, the results of noise and vibration measurements are given in Fig. 6.
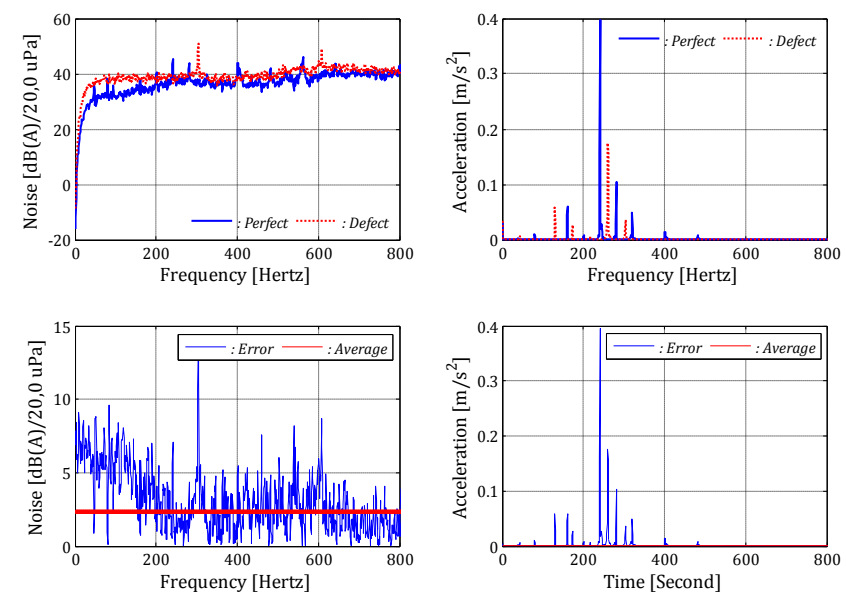

Fig. 6: Noise and vibration measurements for Case 4

In this Case, bearing has a lubrication problem. By evaluating the vibration measurement in Fig. 6, the acceleration values are nearly same for perfect and defect fans. On the contrary, there is a certain difference for noises of perfect and defect fans. So, it is very effective to use the noise measurement to detect this fault type in mechanical systems.

The results of noise and vibration measurements for Case 5 and Case 6 are outlined in Figs. 7 and 8, respectively.
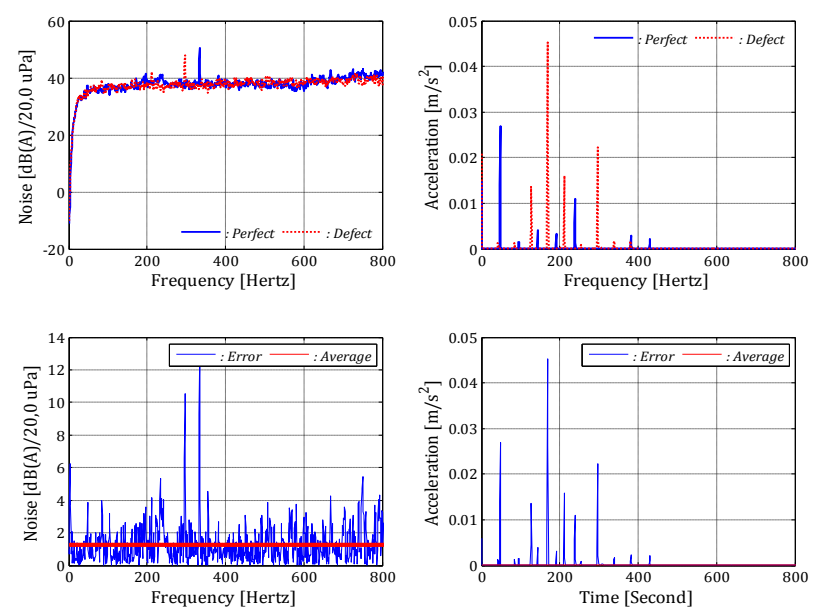

Fig. 7:Noise and vibration measurements for Case 5 

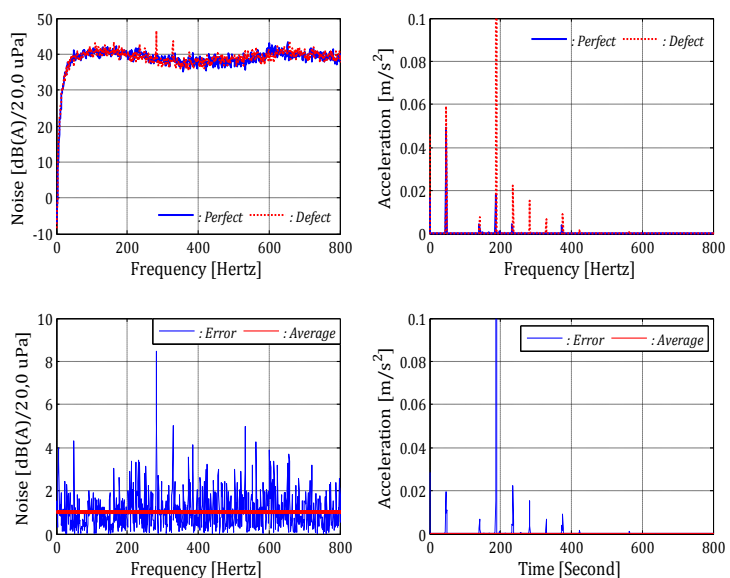

Fig. 8: Noise and vibration measurements for Case 6

As seen from Figs. 7 and 8, there is a clear difference for vibration results of perfect and defect fans. On the contrary, for each case, the noise results are nearly same. So, vibration measurement is very effective to detect these faults types. Numerical values for different cases are outlined in Table 3.

Table 3. Numerical evaluations for different case studies

\begin{tabular}{|c|c|c|c|c|c|c|c|c|c|c|}
\hline & \multicolumn{2}{|c|}{ Crest Factor } & \multicolumn{2}{|c|}{ Kurtosis } & \multicolumn{2}{|c|}{ Average error } & \multicolumn{2}{|c|}{ Max. error } & \multirow{2}{*}{$\begin{array}{l}\text { Max. } \\
\text { freq. } \\
\text { Noise }\end{array}$} & \multirow{2}{*}{$\begin{array}{c}\text { error } \\
\text { Vibr. }\end{array}$} \\
\hline & Noise & Vibr. & Noise & Vibr. & Noise & Vibr. & Noise & Vibr. & & \\
\hline $\begin{array}{l}\text { Case } \\
1\end{array}$ & 16,95 & 21,72 & 10,01 & 312,42 & 1,92 & 0,00026 & 10,02 & 0,0542 & 37 & 32 \\
\hline $\begin{array}{l}\text { Case } \\
2\end{array}$ & 14,08 & 21,87 & 10,07 & 357,69 & 10,4 & 0,00034 & 20,1 & 0,1505 & 37 & 228 \\
\hline $\begin{array}{l}\text { Case } \\
3\end{array}$ & 17,20 & 11,38 & 13,78 & 274,52 & 1,64 & 0,000018 & 8,98 & 0,0067 & 47 & 35 \\
\hline $\begin{array}{l}\text { Case } \\
\mathbf{4}\end{array}$ & 27,17 & 42,73 & 396,0 & 266,0 & 2,43 & 0,00133 & 14,34 & 0,3963 & 304 & 241 \\
\hline $\begin{array}{l}\text { Case } \\
5\end{array}$ & 38,09 & 18,55 & 437,7 & 188,7 & 1,34 & 0,00018 & 13,52 & 0,0453 & 334 & 169 \\
\hline $\begin{array}{l}\text { Case } \\
\mathbf{6}\end{array}$ & 23,38 & 25,55 & 117,08 & 548,9 & 1,05 & 0,00042 & 8,473 & 0,30 & 282 & 187 \\
\hline
\end{tabular}

According the Kurtosis formulation, if the Kurtosis value is bigger than 3 , there is a fault in the system. In this study, each case has an artificial fault. As outlined in Table 3, all Kurtosis values for noise are bigger than 3. On the contrary, these values for vibration are not fully bigger than 3 . So, noise measurement is more sensitive for detecting the faults than vibration in such mechanical systems.

\section{CONCLUSION}

In this study, noise and vibration measurement are implemented to investigate the fan fault problems. As outlined in different case studies, noise measurement in addition to vibration can be used for detecting the faults in system. Particularly, noise measurement is very effective to detect the possible faults in blade problem (near the bearing) and lubrication problem. Another advantage of noise measurement is a contactless measurement. Acoustic sensor can be located everywhere on the system. But, there are some constraints for vibration sensor. It has to be usually located on bearing.

\section{ACKNOWLEDGEMENT}

This work is a part of the research project FBA-12-4111. The authors wish to express their thanks for financial support being provided by the Scientific Research Project Fund of Erciyes University, in carrying out this study.

\section{REFERENCES}

[1] Dr. Géza Husi, Dr. Csaba Szász: Building Automation Technology in Electrical Engineering and Mechatronics Department in Debrecen. 671674 pp. Ulusal Makína Teorisi Sempozyumu, 12-13 Eylül, 2013, Erzurum.

[2] J. Tian, J.J. Gu, X.Z. Peng, Z.M. Qin, “A Fault Diagnosis Method Based on Wavelet Approximate Entropy For Fan", Proceedings of the Sixth International Conference on Machine Learning and Cybernetics, Hong Kong, 19-22 August 2007.

[3] Q. Miao, M. Azarian, M. Pecht, "Cooling Fan Bearing Fault Identification Using Vibration Measurement", 978-1-4244-9827-7/11 C2011 IEEE.

[4] M.A.A. Elmaleeh M. Bin Saad, "A Study of Acoustic Emission Technique on Incipient Detection of Rotating Machine Faults", 10th Int. Conf. on Control, Automation, Robotics and Vision, Hanoi, Vietnam, 17-20 December 2008.

[5] S. Velarde-Suarez, R. Ballesteros-Tajadura, J.P. Hurtado-Cruz, C. Santolarrio-Morros, "Experimental determination of the tonal noise sources in a centrifugal fan", Journal of Sound and Vibration, vol. 295, pp. 781-796, 2006.

[6] S. Velarde-Suarez, R. Ballesteros-Tajadura, C. Santolarrio-Morros, B. Pererias-Garcia, "Reduction of the aerodynamic tonal noise of a forward-curved centrifugal fan by modification of the volute tongue geometry", Applied Acoustics, vol. 69, pp. 225-232, 2008.

[7] S.F. Wu, S.G. Su, H.S. Shah, "Modelling of the noise spectra of axial flow fans in a free field", Journal of Sound and Vibration, vol. 200(4), pp. 379-399, 1997.

[8] L. Lin, W. Lu, F. Chu, "Application of AE techniques for the detection of wind turbine using Hilbert-Huang transform", Prognostics\& System Health Management Conference (PHM2010 Macau), IEEE, 2010

[9] X. Tian, "Cooling Fan Reliability: Failure Criteria, Accelerated Life Testing, Modeling and Qualification", 1-4244-0008-2/06 @2006 IEEE.

[10] Z. Yin, T. Han, J. Wang, "Fan Fault Diagnosis System Based on Virtual Prototyping Technology", Fifth International Conference on Fuzzy Systems and Knowledge Discovery, 978-0-7695-3305-6/08 () 2008 IEEE.

[11] Tandon N., Choudhury A. "A review of vibration and acoustic measurement methods for the detection of defects in rolling element bearings", Tribology International, vol. 32, pp. 469-480, 199.

[12] T.H. Loutas, J. Kalaitzoglou, G. Sotiriades, E. Kayias, V. Kostopoulos, "Diagnosis of Artificial Gear Defects on Single Stage Gearbox using Acoustic Emission", Advanced Material Research, vol. 13-14,pp 415420, 2006.

[13] T. Toutountzakis, C.K. Tan, D. Mba, "Application of acoustic emission to seeded gear fault detection", NDT\&E International vol. 38, pp. 27-36, 2005.

[14] Q. Miao, M. Azarian, M. Pecht, "Cooling Fan Bearing Fault Identification Using Vibration Measurement” C2011 IEEE, 978-1-42449827-7/11.

[15] Brüel \& Kjær Sound \& Vibration Measurement A/S. http://www.bksv.com 